\title{
Effect of Atomic Oxygen Irradiation on Field Emission Cathodes in Low Earth Orbit
}

\author{
By Atsuko ShimadA ${ }^{1)}$, Yoshinobu TAnAKA ${ }^{1)}$, Yasushi OHKAwA $^{2)}$, Koji MATSUmoto $^{2)}$, Masahito TAGAwA ${ }^{3)}$, \\ Makoto MATSUI $^{1)}$ and Yoshiki YAMAGIWA ${ }^{1)}$ \\ ${ }^{1)}$ Shizuoka University, Hamamatsu, Japan \\ ${ }^{2)}$ Japan Aerospace Exploration Agency, Chofu, Japan \\ ${ }^{3)}$ Kobe University, Kobe, Japan
}

(Received June 28th, 2013)

\begin{abstract}
An Electrodynamic tether (EDT) is an attractive propulsion device for active debris removal systems. One of the key components of the EDT system is an electron emission device, and we have studied a field emission cathode (FEC) using carbon nanotubes (CNTs) because of its simplicity and potential capabilities. Since EDT systems are operated in low earth orbit (LEO), the CNTs in the FEC may be affected by atomic oxygen (AO), so the effect of AO irradiation on the FEC was studied. We conducted AO irradiation tests on FECs using laser detonation beam facilities and compared the pre- and post-irradiation electron emission characteristics. As a result, the FEC could not emit electrons when the total AO fluence of $3 \times 10^{20} / \mathrm{cm}^{2}$ was irradiated perpendicular to the emission surface. In this case, CNTs on the emitter surface disappeared by AO irradiation. When the irradiation direction was parallel to the emission surface, on the other hand, the FEC could emit electrons after the irradiation although the required voltage increased up to 1.5 times as high as that of pre-irradiation condition. This comparison indicated that the direction of $\mathrm{AO}$ irradiation has strong effect on the performance degradation of the FEC on orbit.
\end{abstract}

Key Words: Field Emission Cathode, Carbon Nanotube, Atomic Oxygen

\section{Nomenclature}

$\begin{array}{cll}e & : & \text { elementary charge } \\ E & : & \text { electric field } \\ F & : & \text { local electric field } \\ h & : & \text { Plank's constant } \\ J & : & \text { current density } \\ m & : & \text { mass } \\ \beta & : & \text { field enhancement factor } \\ \phi & : & \text { work function } \\ \text { Subscripts } & \\ e & : \text { electron }\end{array}$

\section{Introduction}

The population of space debris in low earth orbit (LEO) will continue increasing, even if no new objects are launched ${ }^{1)}$. In Japan Aerospace Exploration Agency (JAXA), active debris removal systems using electrodynamic tethers (EDTs) have been studied to resolve this space debris problem. An EDT system (Fig. 1) is a promising candidate of de-orbit propulsion for active debris removal systems because of its propellant-free mechanism. Since thrust of the EDT is generated by the interaction between the current via the tether and the geomagnetic field, no propellant is needed in this propulsion system. To drive the electric current through the tether, a closed electrical circuit must be established via the surrounding plasma by collecting electrons at one end and emitting them at the other end of the tether. A field emission cathode (FEC) is a suitable electron emission device in the EDT system because the FEC system can be simple and low-power without working gas or a heater. This FEC uses a carbon nanotube (CNT) as an electron emitter because CNTs have superior mechanical strength and high-aspect ratios.

A debris removal mission will be conducted, orbiting at an altitude of around 1000 to $600 \mathrm{~km}$. When the EDT system operates to remove debris, the higher number density of atomic oxygen (AO) in the low earth orbit (LEO) environment is problematic and means the electron emission performance may decline due to the collision of AO. Gasdaska et al. showed that FEC performance declines in an environment of

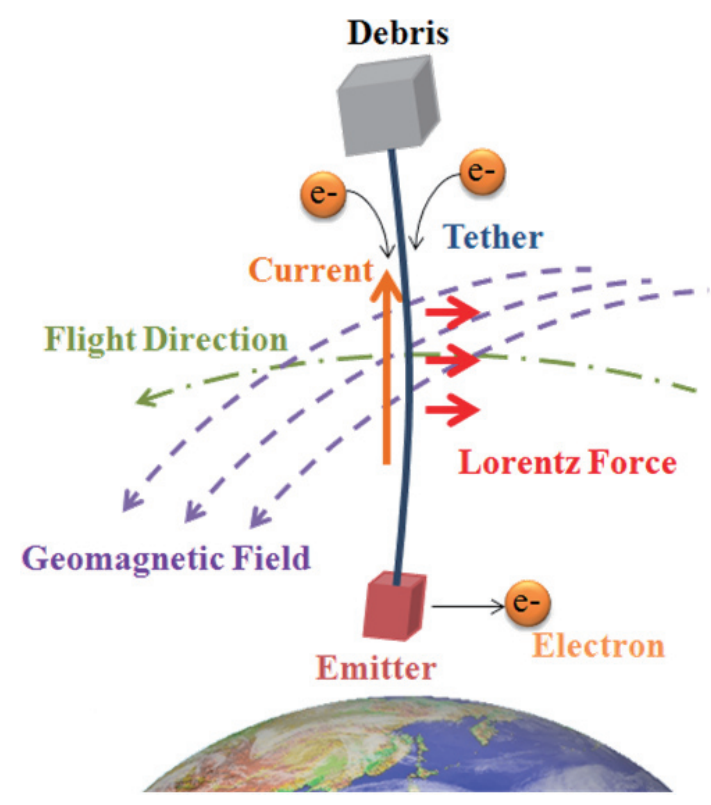

Fig. 1. Conceptual image of the EDT system. 
molecular oxygen ${ }^{2)}$. Another study showed that the field emission performance from single-walled CNTs was susceptible in an oxygen environment compared with those in other gaseous environments such as hydrogen, water vapor and argon ${ }^{3)}$. The experimental environment in these studies was different from the practical orbital environment where AO possesses orbital velocity. Therefore, AO irradiation tests on the FEC should be done for estimating the life of the FEC on orbit.

In this paper, the effect of AO irradiation on the FEC electron emission performance was studied by AO irradiation at an orbital velocity of approximately $8 \mathrm{~km} / \mathrm{s}$. Three cases of irradiation direction (angle of attack) to the FEC emission surface (perpendicular, parallel and 10 degrees) were tested. The post-irradiation current-voltage characteristics for electron extraction were obtained and compared with those before irradiation. In addition, the long-term operation of the FEC and the microscopic observation of the CNT emitters were conducted.

\section{Field Emission Cathode (FEC)}

An FEC is a cathode device using an electron field emission, which is a phenomenon whereby electrons are extracted from emitter material by applying a strong electric field. A conceptual image of the FEC is shown in Fig. 2. When a strong electric field is applied to the emitter surface, the thickness of the potential barrier becomes thin significantly, allowing electrons to pass through via wave characteristics. This is known as a "tunneling effect." For electron emissions, a strong electrical field of about $10^{9} \mathrm{~V} / \mathrm{m}$ on the emitter surface is required. In FEC using CNTs, the electric fields at the tips of CNTs are enhanced by its nano-scale tube diameter.

The current density $J\left[\mathrm{~A} / \mathrm{m}^{2}\right]$ of field emission is described by the Fowler-Noldheim equation:

$$
J=\frac{e^{3} F^{2}}{8 \pi h \phi} \exp \left(-\frac{8 \pi \sqrt{2 m_{e}}}{3 h e F} \phi^{3 / 2}\right)
$$

where $e$ is the electron charge, $h$ Planck's constant, $m_{e}$ the electron mass, $\phi$ the work function of the emission material and $F$ the local electric field. The local electric field $F[\mathrm{~V} / \mathrm{m}]$ at the tips is described by the equation:

$$
F=\beta E
$$

where $\beta$ is the field enhancement factor, $E$ is the electric field between the electrodes.

The FEC advantages include no working gas requirement,

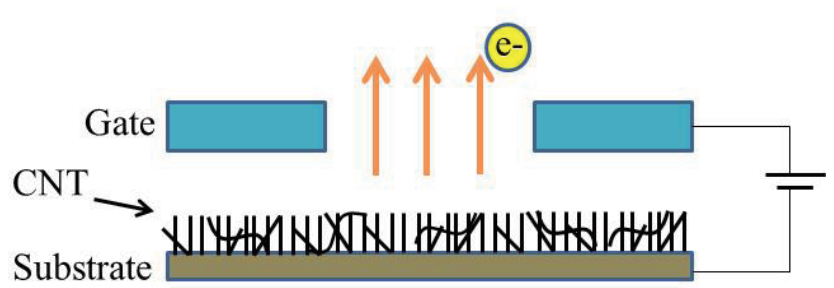

Fig. 2. Conceptual image of the FEC. simplified structure and low power consumption. These characteristics are important applying the FEC to the EDT system for future active debris removal satellites to facilitate cost-effective debris removal systems in a simpler way. The FEC also possesses some disadvantages such as the need for high-voltage power conditioners, the emission current restriction by space-charge, and the decline in performance due to exposure to AO and the plasma environment in LEO. This study was conducted to quantify the influences of the AO environment on FEC characteristics.

\section{Experimental Setup and Procedure}

The current-voltage characteristics in electron emission operation and time-dependent performance change in the long-term operation of the FEC were obtained before and after the AO irradiation. The surface of the CNT emitters was observed using a scanning electron microscope (SEM) and quantitative analysis was done using an electron probe microanalyzer (EPMA). We conducted AO irradiation tests on the FECs using laser detonation beam facilities. The AO velocity was about $8 \mathrm{~km} / \mathrm{s}$, namely equivalent to orbital velocity, which corresponds to translational energy of $5 \mathrm{eV}^{4)}$.

AO irradiation direction to the FEC surface is an important factor to evaluate the in-orbit life of the FEC considering the position of the FEC on spacecraft. In this experiment, we selected three cases of the AO irradiation direction; perpendicular, parallel and 10 degrees relative to the FEC electron emission surface (Fig. 3). These irradiation directions were selected by following reasons. 1) Perpendicular case: for simulating the most damaging condition on orbit when the FEC is located on the ram side of spacecraft. 2) Parallel case: for simulating the smallest damaging condition on orbit when the FEC is located parallel to the orbital velocity. 3) 10-degree case: for simulating the situation that the spacecraft attitude cannot be controlled accurately to keep the parallel condition and possesses a small angle of attack.

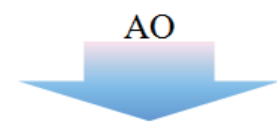

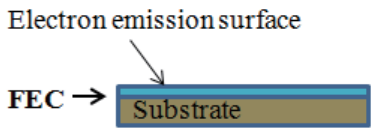

Perpendicular

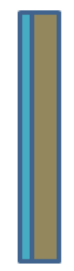

Parallel

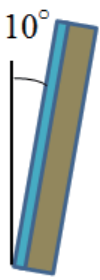

10 degree
Fig. 3. Direction of AO irradiation. 
Table 1. Irradiation condition

\begin{tabular}{|c|c|c|}
\hline Case & Fluence $\left[/ \mathrm{cm}^{2}\right]$ & $\begin{array}{c}\text { Equivalent exposure time in } 800-\mathrm{km} \text {-alt } \\
{[\text { days }]}\end{array}$ \\
\hline $\mathrm{A}$ & $3 \times 10^{20}$ & 1500 \\
\hline $\mathrm{B}$ & $3 \times 10^{19}$ & 150 \\
\hline $\mathrm{C}$ & $1 \times 10^{19}$ & 50 \\
\hline $\mathrm{D}$ & $3 \times 10^{18}$ & 15 \\
\hline
\end{tabular}

Four levels of total AO fluence were selected in the perpendicular irradiation test; A) $3 \times 10^{20}$, B) $3 \times 10^{19}$, C) $1 \times 10^{19}$, and D) $3 \times 10^{18} / \mathrm{cm}^{2}$. These fluences are equivalent to the environments at $800 \mathrm{~km}$ altitude shown in Table 1. In the irradiation tests of parallel and 10 degree directions, the current-voltage characteristics of the FECs were obtained at fluence levels of $3 \times 10^{18}, 1 \times 10^{19}, 3 \times 10^{19}, 1 \times 10^{20}$, and $2 \times 10^{20} / \mathrm{cm}^{2}$. (Here, "Fluence" denotes the accumulated AO flux in a unit area perpendicular to the AO flow and does not depend on the angle of the specimen.)

The overview and schematic drawing of the FEC are shown in Figs. 4 and 5. The FEC consists of a CNT-emitter and mask and gate electrodes. The gate electrode was insulated from the emitter by an insulation spacer. The circuit diagram for the electron emission is shown in Fig. 6. The emitter was grounded and the gate electrode potential was positively swept to obtain the current-voltage characteristics. The emission characteristics changes in long-term operation were evaluated by measuring the variation in the applied extraction voltage required to maintain a constant $1-\mathrm{mA}$ emission current for 50-hours.

Quantitative analyses of the CNT emitter surface were conducted using the EPMA, for five irradiated and non-irradiated sites respectively.

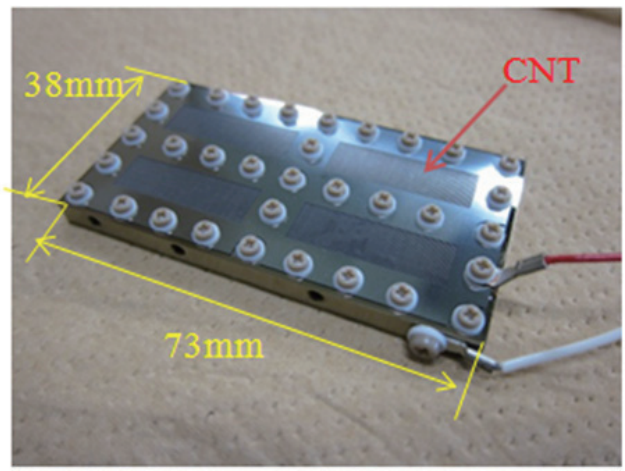

Fig. 4. Photograph of the FEC.

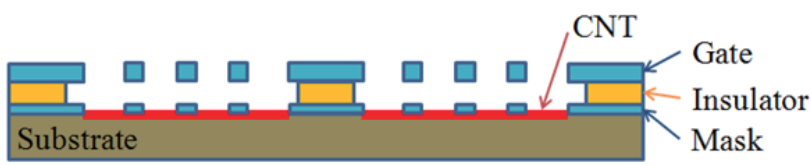

Fig. 5. Schematic drawing of the FEC.

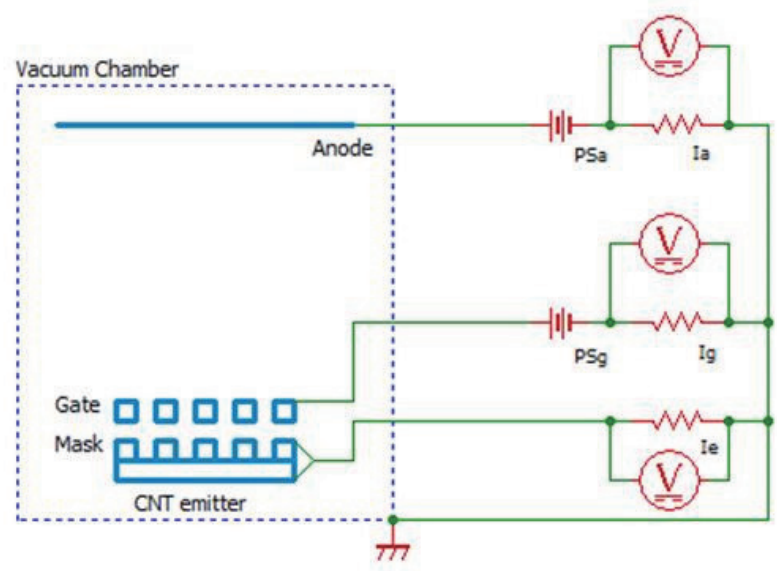

Fig. 6. Experimental circuit diagram for electron extraction.

\section{Experimental Results}

\subsection{Perpendicular irradiation}

Figure 7 shows the current-voltage characteristics before and after AO irradiation for four cases of total fluence. According to Fig. 7, the extraction voltages needed for electron emission increased after AO irradiation in all cases compared with those before the irradiation. The results indicated that the performance degradation was dependent on total AO fluence. In case A, the FEC could not emit electrons even when an extraction voltage of $1000 \mathrm{~V}$ was applied.

Table 2 shows the threshold voltages for $0.01-\mathrm{mA}$ electron emission in all cases, showing that the post-irradiation threshold voltages exceeded those before irradiation. In addition, it was found that the threshold voltage ratio, namely the ratio before and after irradiation, rose with increasing total AO fluences. These results indicated that the performance decline in FEC caused by the AO irradiation was considerable when the irradiation direction was perpendicular to the emitter surface.

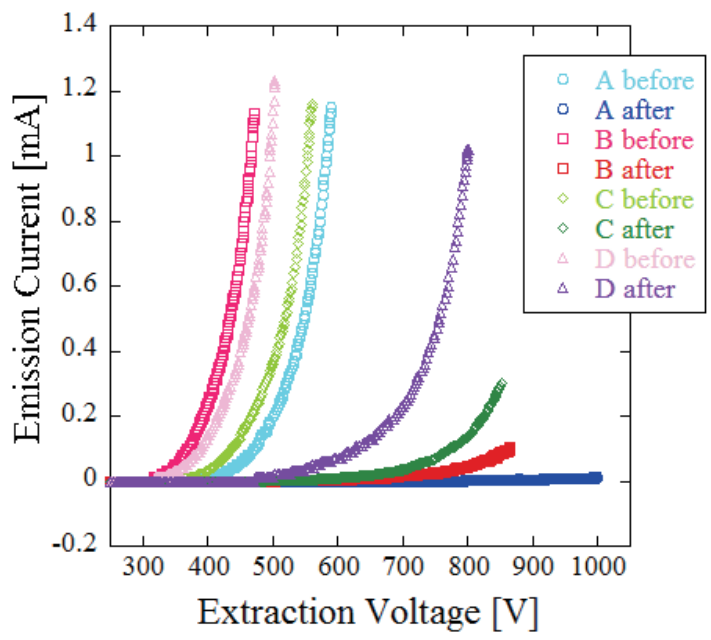

Fig. 7. Emission current vs. extraction voltage before and after perpendicular AO irradiation. Total AO fluences were $3 \times 10^{20}, 3 \times 10^{19}$, $1 \times 10^{19}$, and $3 \times 10^{18} / \mathrm{cm}^{2}$ for Case A, B, C and D respectively. 
Table 2. Threshold voltage for electron emission

\begin{tabular}{|c|c|c|c|c|}
\hline \multirow{2}{*}{ Case } & \multirow{2}{*}{$\begin{array}{c}\text { Fluence } \\
{\left[/ \mathrm{cm}^{2}\right]}\end{array}$} & \multicolumn{2}{|c|}{ Threshold voltage [V] } & \multirow{2}{*}{ Voltage ratio [-] } \\
\cline { 3 - 4 } & $3 \times 10^{20}$ & Before & After & - \\
\hline $\mathrm{A}$ & $3 \times 10^{19}$ & 320 & - & 2.07 \\
\hline $\mathrm{B}$ & $1 \times 10^{19}$ & 370 & 530 & 1.43 \\
\hline $\mathrm{C}$ & $3 \times 10^{18}$ & 320 & 360 & 1.13 \\
\hline $\mathrm{D}$ & & & & \\
\hline
\end{tabular}

\subsection{Parallel irradiation}

To evaluate the influence of the AO irradiation direction on the FEC surface, another experiment was conducted using the AO irradiation test facility of Kobe University. In this test, three FEC samples were used, two of which located parallel to the AO flow and the one with an angle of attack of 10 degrees. Figure 8 shows the extraction voltages required for $1-\mathrm{mA}$ emission at fluence levels of $3 \times 10^{18}, 1 \times 10^{19}, 3 \times 10^{19}, 1 \times 10^{20}$, and $2 \times 10^{20} / \mathrm{cm}^{2}$ for these three cases.

Figure 8 shows that the extraction voltages rose with increasing $\mathrm{AO}$ fluences in all cases, but that the voltage increment was quite moderate compared with that in case of perpendicular irradiation. Another important point is that the increase in extraction voltages was not proportional to the fluence but to its logarithm, which indicates that the performance degradation subsides after a certain level of $\mathrm{AO}$ exposure.

The performance degradation of the FEC in the case of 10-degree direction was more severe than the case of parallel irradiation, but much less than the case of perpendicular irradiation. It was thus noted that the degradation of the FEC's electron emission performance was reduced by locating an emitter surface parallel to the orbital velocity. In addition when the direction of the FEC changed slightly up to 10 degrees, the performance degradation was modest.

Table 3 shows the extraction voltage for $1-\mathrm{mA}$ emission before and after AO irradiation in parallel and perpendicular

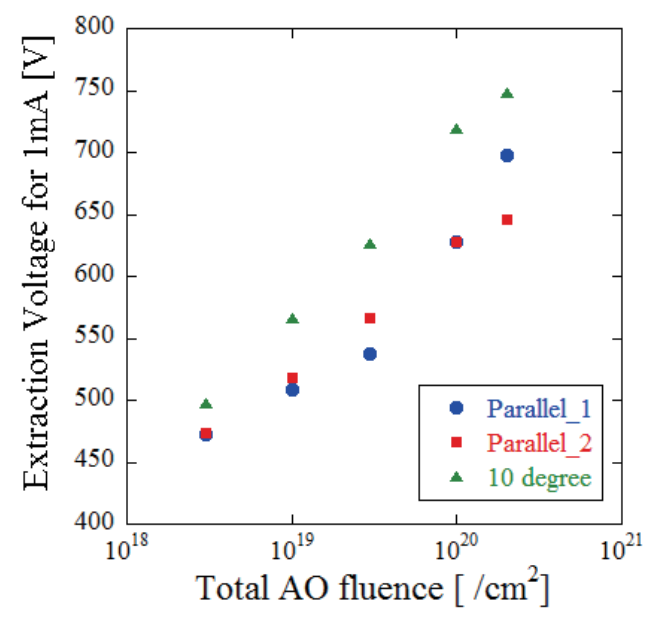

Fig. 8. Extraction voltage required for 1-mA emission vs. Total AO fluence in cases of parallel and 10 degree biased forms of irradiation. Two FECs were located at parallel direction for considering individual variability (one FEC is called "Parallel_1" and the other is "Parallel_2"). cases. In these three cases, the extraction voltage ratios were almost equal at around 1.5 , which indicates that parallel $\mathrm{AO}$ irradiation of $2 \times 10^{20} / \mathrm{cm}^{2}$ resulted in performance degradation almost equivalent or less to the perpendicular irradiation of $3 \times 10^{18} / \mathrm{cm}^{2}$.

Table 3 indicated the performance degradation of FEC in parallel cases was much less than that in case of perpendicular irradiation. A major reason for this difference was the difference in the kinetic energy of AO collision to the emitter surface. In the perpendicular case, AO collides with the CNT-emitter with $8 \mathrm{~km} / \mathrm{s}$, conversely, averaged impingement energy in the parallel case should be quite low compared with the perpendicular case. Although the main reasons for the performance degradation in the parallel case is unclear at present, high velocity AO impingement by the scattering from the anode plate or chamber wall, or physical and/or chemical absorption of oxygen on CNTs are potential candidates.

Table 3. Comparison of the Extraction voltage (for $1 \mathrm{~mA}$-emission) ratio in parallel and perpendicular irradiation cases.

\begin{tabular}{|c|c|c|c|c|}
\hline \multirow{2}{*}{ Case } & \multirow{2}{*}{$\begin{array}{c}\text { Fluence } \\
{\left[/ \mathrm{cm}^{2}\right]}\end{array}$} & \multicolumn{2}{|c|}{ Extraction voltage [V] } & \multirow{2}{*}{$\begin{array}{c}\text { Voltage } \\
\text { ratio [-] }\end{array}$} \\
\cline { 3 - 4 } & $2 \times 10^{20}$ & 423 & 698 & 1.65 \\
\hline Parallel_1 & $2 \times 10^{20}$ & 437 & 646 & 1.47 \\
\hline Parallel_2 & $3 \times 10^{18}$ & 496 & 800 & 1.61 \\
\hline Perpendicular & 390 & After & \\
\hline
\end{tabular}

\subsection{Emission characteristics change in long-term operation}

The change in emission characteristics during long-term operation was evaluated before and after parallel AO irradiation and time dependent changes in the extraction voltage ratio before and after irradiation during 50-hour operation are shown in Fig. 9. Before the AO irradiation, the extraction voltage ratio remained unchanged throughout the experiment, while conversely, the extraction voltage ratio rose over time after the irradiation. This difference in voltage rise may be attributable to chemical or physical interactions by the AO irradiation.

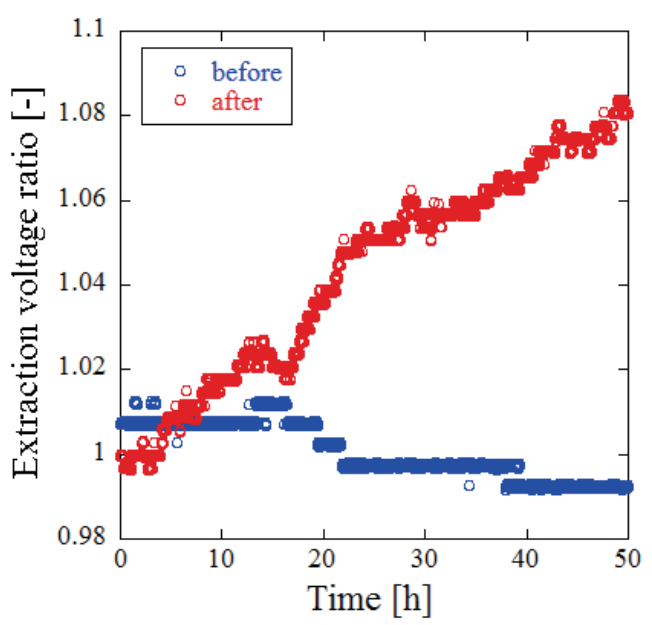

Fig. 9. Extraction voltage ratio before and after parallel AO irradiation vs. elapsed time. Total AO fluence was $2 \times 10^{20} / \mathrm{cm}^{2}$. 


\subsection{Microscopic observation of the CNT emitter surface}

Figure 10 shows an SEM image of the CNT emitter surface following perpendicular AO irradiation of about $3 \times 10^{20} / \mathrm{cm}^{2}$ (Case A). Figure 10(a) is an image of a non-irradiated site, which is below the mask electrode (see Fig. 5), while (b) is that of an irradiated site.

Figure 10(a) shows CNTs which resemble white strings on the substrate, whereas no such, strings are observed in Fig. 10(b). Therefore it was identified that CNTs had disappeared due to AO irradiation. Since no electron emission was observed in Case A, this CNT erosion should be the main reason for the decline in FEC performance.

Quantitative analysis of the emitter surface using EPMA was conducted to verify the above observation results. Figure 11 shows the remaining ratio of carbon, which is calculated by dividing the detected carbon intensities on the irradiated site by those on the non-irradiated site. Since the CNT distribution on the substrate is not homogeneous, the remaining ratio became over $100 \%$ at some sampling points. The results of 25 points of measurement are plotted in this figure as open circle symbols, while the average values are indicated by filled square markers.

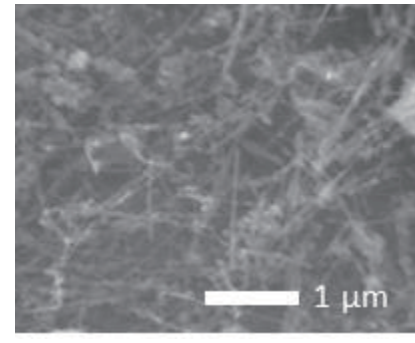

(a)

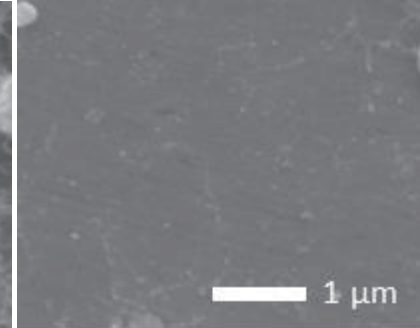

(b)
Fig. 10. SEM image of a CNT emitter surface. (a) non-irradiated site, (b) irradiated site.

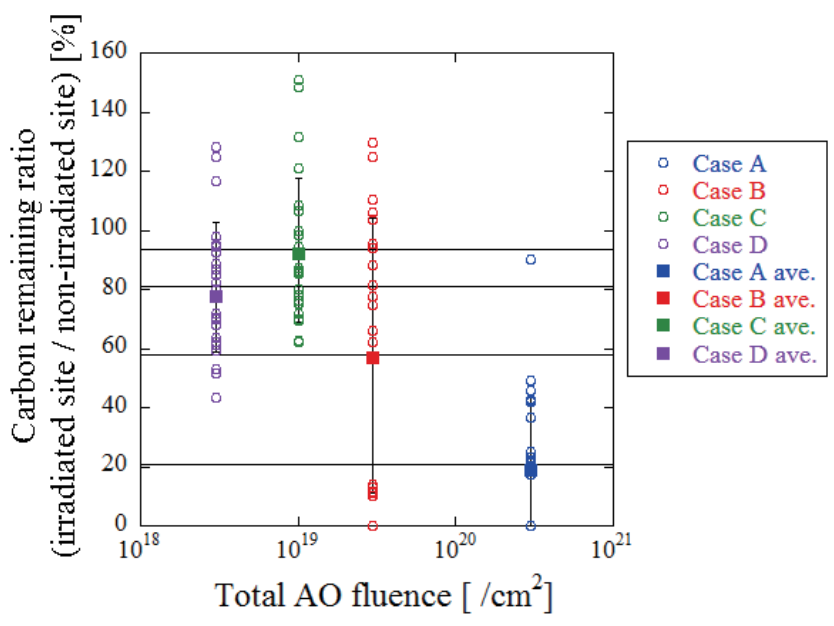

Fig. 11. Remaining ratio of carbon after perpendicular AO irradiation. Total AO fluences were $3 \times 10^{20}, 3 \times 10^{19}, 1 \times 10^{19}$, and $3 \times 10^{18} / \mathrm{cm}^{2}$ for Cases $\mathrm{A}, \mathrm{B}, \mathrm{C}$ and $\mathrm{D}$ respectively.
Although the measurement results vary considerably, Fig. 11 shows a rough trend whereby the carbon remaining ratio rises with decreasing total $\mathrm{AO}$ fluences. In Case $\mathrm{A}$ $\left(3 \times 10^{20} / \mathrm{cm}^{2}\right)$, the remaining ratio is relatively low and consistent with the observation result shown in Fig. 10. Conversely, however, this analysis result also indicates that a small amount of CNT remains on the substrate to some extent, even in Case A and this indication may be inconsistent with the result of the electron emission test shown in Fig. 7, which shows no electron emission detected in Case A. Regarding this disagreement, we guess that the CNTs detected in Case A do not contribute to electron emission because those tubes are damaged or not firmly attached to the substrate due to AO irradiation. More detailed analysis and discussion is required to clarify these phenomena.

\section{Discussion}

A typical altitude change profile in a debris removal operation using EDT was numerically estimated by JAXA ${ }^{5)}$. In this estimation, 10-km-length EDT is used for the removal of a 1400-kg rocket body from 1000-km-altittude and 83-degree-inclination orbit. Expected total AO fluence in this debris removal case is $3.6 \times 10^{19} / \mathrm{cm}^{2}$. As shown in Table 3, FEC degradation by this fluence level can be acceptable when the FEC was set-up at parallel to the orbital motion. So, it is suggested that the FECs can be used as an electron emission device in EDT system. However, the performance degradation of FECs by long-term operation should also be considered for life time estimation. Figure 9 shows that the increasing rate of the extraction voltage ratio after AO irradiation was larger than that in before AO irradiation. Therefore, in the future works, the performance degradation of FECs as a device in debris removal systems should be evaluated considering preand post-AO irradiation and the long-term operation.

\section{Conclusion}

Atomic oxygen irradiation tests were conducted to evaluate the endurance performance of carbon nanotube field emission cathodes in a LEO environment. Atomic oxygen velocity was set at around $8 \mathrm{~km} / \mathrm{s}$ and total fluence was in the order of $10^{20} / \mathrm{cm}^{2}$ simulating the LEO environment. The irradiation direction was varied through perpendicular, parallel, and 10 degrees. The electron emission characteristics were considerably degraded by irradiation when the irradiation angle was perpendicular to the emitter surface and the disappearance of CNT on the substrate was observed. When the atomic oxygen flow was parallel to the emitter surface, conversely, the damage to the cathode was much less than the perpendicular case. Microscopic observation and analysis showed that the remaining ratio of carbon on the substrate was roughly dependent on the total fluence of atomic oxygen irradiation. And, it is suggested that the FECs can be used as 
an electron emission device in EDT system when the FECs are set up at parallel side. However, the performance degradation of FECs by long-term operation should also be considered.

\section{References}

1) Liou, J. C. and Johnson, N. L.: Risks in Space from Orbiting Debris, Science, 311 (2006), pp.340-341.

2) Charles, J. G. et al: Testing of Carbon Nanotube Field Emission
Cathodes, AIAA, 2004, AIAA 2004-3427.

3) Dean, K. A. and Chalamala, B. R.: The environment stability of field emission from single-walled carbon nanotubes, Appl. Phys. Lett., 75 (1999), pp.3017-3019.

4) Shimamura, H., Baba, S. and Miyazaki, E.: Handbook of the Combined Space Effect Test Facility, JAXA Research and Development Memorandum, 2011, JAXA-RM-10-013.

5) Kawamoto, S., Ohkawa, Y., Kitamura, S. and Nishida, S.: Strategy for active debris removal using electrodynamic tether, Trans. JSASS Space Tech. Japan, 7, ists26(2009), pp. Pr_2_7-Pr_2_12. 